Documentation et bibliothèques

DOCUMENTATION BIBLIOTHËQUES

\title{
Les tendances de la recherche sur les habitudes de lecture au Québec
}

\section{Lise Brunet}

Volume 26, numéro 3, septembre 1980

URI : https://id.erudit.org/iderudit/1054229ar

DOI : https://doi.org/10.7202/1054229ar

Aller au sommaire du numéro

\section{Éditeur(s)}

Association pour l'avancement des sciences et des techniques de la documentation (ASTED)

\section{ISSN}

0315-2340 (imprimé)

2291-8949 (numérique)

Découvrir la revue

Citer cet article

Brunet, L. (1980). Les tendances de la recherche sur les habitudes de lecture au Québec. Documentation et bibliothèques, 26(3), 161-167.

https://doi.org/10.7202/1054229ar

Tous droits réservés (C) Association pour l'avancement des sciences et des techniques de la documentation (ASTED), 1980
Ce document est protégé par la loi sur le droit d'auteur. L’utilisation des services d'Érudit (y compris la reproduction) est assujettie à sa politique d'utilisation que vous pouvez consulter en ligne.

https://apropos.erudit.org/fr/usagers/politique-dutilisation/ 


\section{chronique de larecherche}

\section{Les tendances de la recherche sur les habitudes de lecture au Québec}

Dans le texte de sa communication au premier congrès de la Société française des sciences de l'information et de la communication tenu à Compiègne en avril $1978^{1}$, Nicole Robine s'inquiète de la prolifération des enquêtes sur la lecture en France: plus de 150 depuis 1955. II ne semble pas que nous en soyons là au Québec. C'est pourquoi, lorsqu'il y a quelques mois l'Éditeur officiel du Québec publiait une recherche commandée par le ministère des Affaires culturelles au Centre de recherche sur l'opinion publique (CROP), recherche dans laquelle une large part est faite à la lecture parmi les activités culturelles des Québécois, on aurait plutôt eu envie de s'exclamer: enfin une grande enquête effectuée sur un échantillon national représentatif! ${ }^{2}$ Aucune enquête de cette ampleur ne trouvait place dans le bilan présenté par Marc Alain dans son article de $1972^{3}$. II s'était donné comme tâche, à l'occasion de l'Année internationale du livre, de faire la revue des études psychosociologiques sur les habitudes de lecture au Canada effectuées entre 1965 et 1972.

Depuis cette date, on peut noter que la recherche sur les habitudes de lecture,

1. Nicole Robine, Pour une unification des champs de recherche sur la lecture, $13 \mathrm{p}$. (texte polycopié).

2. Camille Delude-Clift, Le comportement des Québécois en matière d'activités culturelles de loisir, Québec, Éditeur officiel, 1980, 86 p.

3. Marc Alain, "Recherches psychosociologiques sur les habitudes de lecture au Canada", Bulletin de l'A.C.B.L.F., vol. 18, no 3 (septembre 1972), 191-197. du moins au Québec, attire de plus en plus de chercheurs. Elle se diversifie et il devient possible de caractériser les différentes recherches et même d'en établir une certaine typologie. Des comparaisons peuvent s'établir entre les résultats obtenus par différentes enquêtes et il faut noter la place plus large accordée au lecteur adulte dans cette perspective de recherche.

Depuis la rétrospective de Marc Alain, notre connaissance des habitudes de lecture au Québec s'est accrue grâce à quelques enquêtes nationales et régionales auxquelles nous nous attarderons assez longuement, vu leur importance. Outre ces enquêtes portant sur une large tranche de population, des études se sont limitées à des groupes sociaux plus restreints. II s'agit de groupes composés soit de lecteurs et de non-lecteurs, soit de lecteurs seulement, que ces lecteurs forment ou non la clientèle d'une bibliothèque ou d'un service documentaire. Certaines études se sont attachées à révéler les comportements particuliers aux groupes caractérisés par leur âge, leur occupation professionnelle, leur situation sociale. Quelques travaux apportent des indications sur le lecteur en tant que consommateur d'une source d'information ou d'un genre de lecture alors que d'autres, qui se situent surtout dans le secteur de l'évaluation, étudient le lecteur en tant qu'utilisateur d'un service.

\section{Les Québécois et la lecture}

L'enquête de CROP déjà mentionnée 
se donnait comme objectif de tracer le profil des Québécois en matière d'activités culturelles. II s'agit d'un sondage effectué auprès de la population de langue française et de langue anglaise du Québec. Selon cette enquête, la lecture arrive en deuxième place parmi les activités de loisir préférées des Québécois, après le sport et les autres activités physiques, la radio et la télévision n'arrivant qu'en quatrième choix, après les activités de plein air. Cela peut paraître étonnant. Mais attention, il s'agit de l'affirmation d'une préférence et non d'une mesure appliquée à une pratique réelle; de plus, on ne précise pas le genre de lecture. On peut voir ici une illustration des limites de l'enquête de type sociologique quand il s'agit d'activités valorisantes.

L'enquête révèle encore que près du quart de la population se présente dans une bibliothèque une seule fois par an, tandis que $17 \%$ y vont au moins trois fois par an. On apprend aussi que $13 \%$ de la population visite un salon du livre au cours de l'année et que les trois quarts des Québécois lisent les journaux très ou assez souvent. Par ailleurs, un peu plus de la moitié lit des revues ou magazines et des livres de façon habituelle, les revues préférées étant les magazines d'actualité. Le quart des interviewés a lu au moins un livre par semaine et autant en ont lu une dizaine dans l'année, ce qui donne $50 \%$ de vrais lecteurs.

Quant aux motivations à la lecture, on lit surtout livres et revues par plaisir, dans une proportion moins importante pour passer le temps et, chez une plus faible proportion de gens, pour des études ou le travail. Les personnes qui lisent peu donnent comme principale raison le manque de temps. Les autres raisons mentionnées sont le prix des livres, la difficulté d'accès aux librairies, le manque d'intérêt.

Pour ce qui est de la façon de s'approvisionner en lecture, parmi les lecteurs assidus, $71 \%$ achète ses livres, $26 \%$ les emprunte dans les bibliothèques ou d'amis et de parents. La moitié de ces emprunteurs s'adresse aux bibliothèques publiques et $3 \%$ fréquente une bibliothèque d'enseignement ou s'approvisionne au travail. Seulement $1 \%$ des lecteurs de revues trouve cette littérature dans une bibliothèque et presqu'autant de revues sont acquises par achat que par abonnement. Les préférences des lecteurs sont, par ordre décroissant, les romans, les ouvrages spécialisés ou documentaires sur le bricolage, la cuisine, l'artisanat, etc., les biographies, la littérature classique, la poésie et divers autres sujets.

Cette enquête est complétée par diverses données d'ordre démographique tels la langue maternelle, le sexe, le groupe d'âge, le lieu de résidence de la population interrogée.

Les nombreuses données quantitatives fournies par cette enquête étant obtenues par sondage, elles contiennent une certaine part de subjectivité puisqu'elles reposent sur les affirmations des personnes questionnées. On sait par ailleurs que la méthode statistique permet un certain contrôle de la marge d'erreur lorsque la quantité de sujets est suffisante. II s'agit toutefois de données presque brutes qui demandent analyse, mise en relation, interprétation et peuvent être largement utilisées par la suite au cours de recherches sectorielles. L'intérêt de ces données réside cependant surtout dans le fait que c'est à peu près le seul genre d'enquête où il soit possible de rejoindre à la fois les lecteurs et les non-lecteurs, les usagers et les non-usagers de bibliothèques. L'utilisation des statistiques officielles est à cet effet fort utile. Nul organisme autre qu'un gouvernement n'a les moyens financiers d'enquêter, dans un domaine si peu rentable, sur de vastes populations.

\section{Lecteurs québécois et lecteurs canadiens}

Statistique Canada publie régulièrement des statistiques sur l'édition, la vente du livre et les bibliothèques (statistiques de la culture). En 1975, le Secrétariat d'État a de plus subventionné une enquête particulièrement intéressante sur la lecture comme activité de loisir ${ }^{4}$. Cette enquête, dont l'analyse a été poursuivie jusqu'à tout récemment, s'insérait dans le cadre de celle

4. Rolf E. Schliewen, Les loisirs au Canada 1975, Ottawa, Secrétariat d'État, 1977, 192 p. 
qui est menée tous les mois sur le travail de la population active. Elle permet entre autres d'établir des comparaisons entre les habitudes de lecture des Canadiens des différentes provinces. On constate par exemple par les résultats de cette enquête qu'on consacre moins de temps à la lecture au Québec qu'en. Colombie britannique. Une explication est proposée à cette donnée statistique, à savoir que les lecteurs de Colombie britannique ne lisent pas tellement plus que les lecteurs du Québec, mais qu'ils sont proportionnellement beaucoup plus nombreux qu'au Québec. On constate de la même façon que l'ouvrier et le col bleu, lorsqu'ils lisent, n'y consacrent pas tellement moins de temps que le professionnel et l'étudiant. En revanche, il y a beaucoup moins d'ouvriers et de cols bleus qui lisent d'où, pour l'ensemble, une moyenne de temps assez faible.

Les Canadiens qui lisent, quel que soit le groupe auquel ils appartiennent, hommes ou femmes, jeunes ou plus âgés, anglais, français ou autres, ouvriers ou professionnels, disent y consacrer en moyenne environ une heure par jour. Les lecteurs ne diffèrent donc pas tellement de l'un à l'autre. Par contre, selon le groupe, ils sont plus ou moins nombreux à le faire; c'est ici surtout que les différences sont appréciables.

Une constatation intéressante de cette enquête, c'est la place qu'occupe la lecture parmi d'autres activités de loisir, comme le signale Yvon Ferland dans son analyse de cette enquête. "Il semble donc que plus la lecture intéresse les Canadiens, plus aussi les autres activités qu'on pourrait qualifier de culturelles les attirent." 5 Sans aller jusqu'à conclure à une relation de cause à effet, on peut cependant constater des liens évidents entre les différents loisirs culturels.

\section{Lecture et télévision}

On a cru pendant un certain temps que la télévision ferait disparaître la lecture. On a d'ailleurs pu constater les premiers signes de ce remplacement d'activités dans la distribution du temps libre. On dis-

5. Yvon Ferland, "La lecture au Canada", Revue Statistique du Canada, (avril 1977), 158. tingue cependant de mieux en mieux la place réelle tenue par la lecture et la télévision parmi les activités de loisir. Cette analyse s'enrichit du fait qu'on tente d'établir des distinctions entre les lecteurs et les non-lecteurs et des catégories parmi les lecteurs, selon l'intensité de lecture et les préférences quant aux media, formes, genres de lecture.

Michel Vastel remarque par exemple que les Québécois qui consacrent environ une heure par jour à la lecture "regardent presque autant la télévision et semblent écouter encore davantage la radio et surtout des disques. Ils sont, en outre, très friands de journaux et de revues, et passent deux fois plus de temps à la pratique d'un hobby que les non-lecteurs"'6.

On pourrait donc croire que le temps passé devant la télévision n'est pas du temps irrémédiablement perdu pour la lecture. On sait pertinemment que, du moins parmi les lecteurs, une activité culturelle est souvent un stimulant qui mène vers d'autres types d'activités culturelles. La vente de livres ou la demande en bibliothèque suscitées par une série télévisée, par la présentation d'une émission littéraire, par un film à succès sont révélatrices à ce sujet.

\section{Données comparables}

Les deux enquêtes nationales susmentionnées, celle du Secrétariat d'État et celle du ministère des Affaires culturelles, offrent évidemment toute une gamme de réflexions et se prêtent particulièrement bien à des exercices de comparaison, notamment à des comparaisons interculturelles. On pourrait par exemple en comparer les résultats avec les données obtenues lors d'un récent sondage fait en France par l'Institut Louis-Harris (la dernière à cette date dans la série des grandes enquêtes nationales) et qui permettait à L'Express de titrer un article "La France se met à lire" ". On apprend dans cet article que le nombre des Français qui lisent a augmenté de $15 \%$ en vingt ans, que ce sont les jeunes qui lisent de plus en plus ainsi

6. Michel Vastel, "On demande des lecteurs", Antennes, 71 (1977), 14

7 J. Jossin et N. Loriot, "La France se met à lire", L'Express, no 1426 (11 novembre 1978), 76-84. 
que les femmes et cela en dépit du fait que pendant les mêmes vingt années, le nombre de postes de télévision soit passé de un à quinze millions. Cependant, on constate aussi quie si la lecture a résisté à la télévision, ce n'est qu'en se transformant considérablement et que si les Français lisent de plus en plus, c'est aussi parce qu'ils lisent autrement.

Il est intéressant de constater que les conclusions d'enquêtes effectuées sur des vastes populations se confrontent assez bien avec celles menées sur des groupes plus restreints, lorsqu'il s'agit des habitudes de lecture.

Bien qu'il n'ait compilé que 140 questionnaires, Alain Bergeron, dans son étude sur les habitudes de lecture des Québécois effectuée en 1973, affirmait déjà la forte population de grands lecteurs chez les jeunes et les femmes ${ }^{8}$. Rappelons qu'il s'agit ici d'une recherche sociologique remarquable par sa rigueur méthodologique et sa richesse dans l'analyse des résultats. Le sociologue Alain Bergeron précisait qu'il ne tenait compte que de la lecture de loisir et qu'il se limitait à un type de lecture, le livre, et à un seul genre, le roman.

\section{Étude des besoins}

II faudrait sans doute rapprocher, dans une typologie des recherches sur la lecture, les grandes enquêtes nationales de celles qui sont faites dans le but d'évaluer les habitudes et besoins documentaires de la population d'une région géographique en tant que futur utilisateur d'une bibliothèque. Le rapport Aubry-Denis, qui se situe dans le secteur des bibliothèques publiques, constitue en quelque sorte une étude de marché: détermination du profil de la clientèle et prévision de sa demande ${ }^{9}$. On sait que les données établies par ce rapport devaient servir de fondement à l'établissement d'un réseau coordonné pour les bibliothèques des

8. Alain Bergeron, Les habitudes de lecture des Québécois, Québec, Institut supérieur des sciences humaines, 1973, $64 \mathrm{p}$.

9. Claude Aubry et Laurent G. Denis, Rapport de l'étude des bibliothèques publiques de la région de Montréal, Québec, Ministère des Affaires culturelles, Service des bibliothèques publiques, $1976,280 p$. municipalités de la Communauté urbaine de Montréal, d'où l'importance d'une étude des composantes de la population. II est entendu que ce rapport s'apparente davantage à un document administratif si on le compare à une étude comme celle de Gilbert Gagnon dont un des buts était également d'identifier la clientèle d'un réseau de bibliothèques publiques ${ }^{10}$. Le rapport Aubry-Denis est cependant davantage orienté vers un examen des structures et est conçu dans une optique de gestion et de planification. II n'en apporte pas moins un éclairage très appréciable sur des réalités démographiques qu'il ne faut pas sous-estimer au niveau de l'étude de milieu.

\section{Lecture adaptée à l’âge}

L'échantillon retenu pour les fins d'une enquête constitue une caractéristique importante de toute recherche. Quand il s'agit de recherche sur les habitudes de lecture, cet échantillon peut aller de l'ensemble de la population à la seule clientèle d'une bibliothèque.

Les travaux de Klara Szpakowska portent directement sur la clientèle d'une bibliothèque. Depuis 1972, madame Szpakowska a publié deux rapports de recherche qui se situent d'emblée parmi les études sur la lecture ${ }^{11}$. Ces recherches se limitent à une clientèle d'un groupe d'âge particulier, les adolescents, les jeunes adultes, et ont comme objectif d'établir un modèle de développement pour une biblio. thèque adaptée à ses usagers.

La majorité des adolescents qui font l'objet des enquêtes de madame Szpakowska ont, il est vrai, une occupation identique: ils sont étudiants. Mais les données qu'elle recueille contribuent

10. Gilbert Gagnon, Habitudes et comportements des lecteurs. Région de la Mauricie, Québec, Ministère des Affaires culturelles, Service des bibliothèques pubiiques, $1970,119 \mathrm{p}$.

11. Janina-Klara Szpakowska, Ces lectures sauvages; panoramique sur le service de bibliothèque aux jeunes adultes, Montréal, Université de Montréal, École de bibliothéconomie, 1972, 222 p.

Besoins en information et en services documentaires chez les jeunes de 14 à 19 ans; sondage réalisé à la Bibliothèque municipale de Ville Saint-Laurent, Montréal, Université de Montréal, École de bibliothéconomie, 1978, 183 p. 
davantage à faire connaître les adolescents comme groupe social que comme groupe professionnel, consommateur d'information et de lecture.

\section{Profil documentaire}

Une étude sur un groupe professionnel ou un milieu spécialisé, celle publiée par Jean de Bonville, porte sur les sources d'information et les habitudes documentaires des journalistes de la presse francophone du Québec ${ }^{12}$. II s'agit d'une enquête effectuée auprès d'un échantillon de journalistes dans le but de dresser le profil documentaire de ce groupe professionnel.

Permettons-nous d'ouvrir ici une courte parenthèse pour justifier l'inclusion de cette étude et de quelques autres parmi les recherches sur les habitudes de lecture. Doit-on en effet séparer les recherches sur les habitudes de lecture et celles sur les habitudes documentaires? Les chercheurs qui s'intéressent à la lecture comme phénomène psychosocial se départagent apparemment d'une manière assez nette et on peut croire que cela tient à ce que la notion de lecture demeure encore imprécise dans ce champ de recherche.

Bien que l'on s'entende généralement pour définir le lecteur comme celui qui lit au moins un livre par mois, on ne distingue pas toujours les niveaux de lecture, c'est-à-dire les motivations à la lecture.

On peut lire simplement avec l'intention de passer le temps, de se détendre, de se divertir. On peut lire avec un but beaucoup plus précis, celui de connaître, d'apprendre ou de s'informer. La lecture se situe alors soit dans une perspective de culture ou de développement personnel, soit dans une perspective de rentabilité, d'efficacité liée à l'occupation professionnelle.

12. Jean de Bonville, Le journaliste et sa documen tation: sources d'information et habitudes documentaires des journalistes de la presse quotidienne francophone du Québec, Québec, EdiGric, 1977, 236 p.

\section{Habitudes de lecture et habitudes documentaires}

Quand on fait l'analyse de la recherche sur le lecteur, la lecture de loisir et la lecture professionnelle apparaissent comme deux pôles opposés, ou du moins comme appartenant à deux univers. S'agitil toujours de la coupure entre le monde de la documentation et celui des bibliothèques? L'expression "spécialiste de I'information documentaire"' (S.I.D.) exprime une volonté de faire disparaître, ou du moins de rétrécir ce fossé. Il faut sans doute voir un effort dans ce sens dans le choix du thème du prochain congrès de l'ASTED centré sur le service aux clientèles, sur l'accès au marché de la lecture et de l'information.

Un autre pas dans ce sens à mentionner est l'introduction dans le programme de maîtrise de l'École de bibliothéconomie de l'Université de Montréal des cours sur le milieu et l'usager dans lesquels la psychosociologie de la lecture et de l'information est mise de l'avant.

\section{L'activité documentaire}

L'étude de Jean de Bonville déjà citée se situe nettement dans la perspective de la lecture professionnelle. De la même façon que lorsque, dans d'autres enquêtes, on tentait d'établir la place occupée par la lecture parmi les activités culturelles, ici on arrive à situer l'activité documentaire parmi les autres activités professionnelles et à mesurer le temps consacré à cette activité, soit environ le tiers du temps de travail.

Jean de Bonville dresse de plus un tableau des sources d'intormation docu. mentaire utilisées par le journaliste de même que celles qu'il n'utilise pas. L'interprétation de ce tableau indique que les sources écrites d'information courantes occupent une place beaucoup plus importante que les sources rétrospectives d'information dans le choix des journalistes.

Quel est le temps consacré à l'activité documentaire, quels sont les sources d'information sélectionnées: ce sont là des indications précieuses sur les habitudes 
documentaires d'un groupe professionnel particulier qui peuvent contribuer à identifier les besoins d'information de ce groupe. Elles révèlent entre autres la faible dextérité documentaire du journaliste et expliquent sans doute la perception sommaire qu'il a du centre de documentation de son journal, bien qu'une majorité l'estime essentiel à son travail.

\section{Étude de clientèle}

Si l'étude de Jean de Bonville appartient à la catégorie des recherches sur les milieux spécialisés professionnels, elle pourrait également être vue dans une certaine mesure comme l'étude de la clientèle actuelle ou potentielle d'une bibliothèque spécialisée. Elle pourrait, en effet, permettre une bonne évaluation des services offerts par le centre de documentation d'un journal, compte tenu des besoins exprimés par ses usagers.

Nous soulevons ici le problème de classification ou de caractérisation des recherches dont la solution tiendrait sans doute dans l'analyse de la finalité de ces différentes recherches et de leur utilisation éventuelle. On pourrait ainsi se demander à quelle catégorie appartient l'étude d'André Castonguay sur Bordeaux ${ }^{13}$. Les usagers de la bibliothèque de la prison de Bordeaux appartiennent à un groupe social particulier, à un milieu dont la spécificité est la marginalité, le milieu carcéral. On ne peut cependant nier que les caractéristiques de ces usagers sont bien identifiables de même que leurs habitudes de vie communes.

\section{Évaluation institutionnelle}

Cependant, cette étude est davantage axée sur une évaluation institutionnelle et concerne plutôt l'organisation fonctionnelle d'une bibliothèque. Elle est néanmoins précieuse dans le cadre de la recherche sur les habitudes de lecture car elle contient de nombreuses données qui pourraient éventuellement être récupérées au niveau de l'analyse psychosociologique.

13. André Castonguay, Lire à Bordeaux, Montréal, Université de Montréal, École de bibliothéconomie, 1973, $127 \mathrm{p}$.

\section{Étude de la satisfaction}

On peut enfin mentionner dans cette revue des recherches sur les habitudes de lecture, toujours dans la perspective de la lecture professionnelle, un dernier type de recherche qui consiste à évaluer un service en vérifiant la satisfaction qu'en tirent ses utilisateurs. À ce genre appartient une recherche faite sur l'utilisation de BADADUQ (Banque de données à accès direct de I'Université du Québec) ${ }^{14}$. Cette étude sur les utilisateurs d'un système de repérage en mode dialogué se rapproche davantage d'une forme d'évaluation institutionnelle que d'une recherche psychosociologique. Elle apporte des informations sur les réactions des usagers compte tenu de leurs attentes vis-à-vis un service documentaire. On y souligne entre autres les réactions émotives des usagers d'un système interactif qui suppose une bonne maîtrise de la machine et certaines aptitudes pour manipuler des concepts logiques. L'impact psychologique des exigences intellectuelles d'une stratégie de recherche est ici mis en lumière. On essaie de mesurer la frustration et même le taux de tolérance à la frustration de l'usager face à un système informatisé, lors de l'interface usager/système. On souligne l'importance en même temps que la méconnaissance actuelle des facteurs humains qui interviennent dans la confrontation de l'usager avec un appareillage informatisé.

À la même catégorie de recherche évaluation d'un service - appartient la recherche publiée très récemment par Raymond Blais sur l'utilisation de RADAR ${ }^{15}$ $L$ 'intention de l'auteur était de vérifier quel rôle joue ce répertoire comme outil de promotion des revues québécoises. Évidemment, les données recueillies sur les usagers dans cette enquête qui en est une sur la satisfaction sont directement liées à l'utilisation d'un service. Cette étude ne se situe sans doute pas au coeur de la recherche psychosociologique sur la

14. Madeleine Balcer et Jean-Paul Gonin, "Réactions de l'usager face à l'utilisation du système de repérage en mode dialogué BADADUQ', Documentaliste, vol. 16, no 2 (mars-avril 1979), 55-61.

15. Raymond Blais, Radar comme outil de promotion et d'utilisation des revues québécoises, Montréal, Bibliothèque nationale du Québec, 1979, 103 p. 
lecture. Elle y ajoute cependant des informations précieuses sur les comportements et les habitudes documentaires permettant éventuellement de préciser les besoins des usagers actuels et potentiels.

\section{Conclusion}

Ce panorama de la recherche sur les habitudes de lecture au Québec ne prétend pas en faire l'inventaire, mais veut plutôt susciter la réflexion quant aux directions que prend cette recherche.

Marc Alain avait mentionné dans son article les difficultés rencontrées pour mettre la main sur les recherches sur la lecture. On pourrait ajouter une difficulté parfois même à les identifier. Nombreuses sont les données sur la lecture accumulées depuis l'Année internationale du livre, mais une bonne quantité de ces données se trouve contenue dans des rapports administratifs, des documents techniques, des relevés statistiques et peut échapper au chercheur.

De plus, la lecture est un phénomène qui peut être abordé sous de multiples aspects, avec des approches scientifiques ou méthodologiques diverses, selon le domaine d'activité ou de spécialisation du chercheur et compte tenu de la finalité de ses recherches. Nombreux sont les intervenants dans le grand processus de la communication de l'information, même si on ne se limite qu'à un seul médium de communication, l'écrit.

La lecture consiste également en un processus individuel intime qui coïncide avec un modèle d'apprentissage, des habitudes, des comportements, des perceptions, des besoins et attentes. Ce processus est mal connu et on est sans doute encore loin d'avoir trouvé des réponses aux questions que l'on se pose entre autres sur les effets de la lecture sur le lecteur.

\section{Lise Brunet}

École de bibliothéconomie Université de Montréal 